\title{
Muon Capture At Large Energy Transfer
}

Article in Physics Letters B · August 1977

Impact Factor: 6.13 · DOI: 10.1016/0370-2693(77)90634-7 · Source: OAI

CITATIONS

36

3 authors, including:

Torleif Ericson

CERN

180 PUBLICATIONS 4,294 CITATIONS

SEE PROFILE
READS

6 


\author{
J. Bernabéu ${ }^{*}$, T.E.O. Ericson and C. Jarlskog ${ }^{+}$) \\ CERN - Geneva
}

\title{
$A B S T R A C T$
}

\begin{abstract}
A novel and parameter free approach to $\mu$ capture is introduced. The inclusive capture rate at maximal energy transfer is connected with the off-mass shell $s$ and $p$ wave scattering lengths for pions. Inside PCAC a rigorous lower bound for the rate results. The vector current contributions are shown to be insignificant. In a numerical illustration it is shown that this picture qualitatively explains the high energy nucleons emitted in $\mu$ capture.
\end{abstract}

\footnotetext{
*)

On leave of absence from Departamento de Física Teórica, Universidad de Barcelona, Spain.

+ )

Present address : Physics Department, University of Bergen, Norway.
}

Ref.TH.2301-CERN

30 March ${ }^{-1} 1977$ 
In the past few years it has been observed that nuclear $\mu$ capture can give neutrons and protons of surprisingly high energy 1). This cannot easily be understood within the usual theory. There exist several deep relations between $\mu$ capture and pion processes. In this paper we will exploit these relations non-trivially to show that pions play a particularly striking rôle at large energy transfer. It will therefore be natural to see the high energy nucleons as a direct indication of the strong relation between $\mu$ capture and pion absorption.

Nuclear $\mu$ capture $\mu^{-}+A \rightarrow \nu+X$ excites mostly nuclear states of low energy. The kinematics is then nearly the same as for $\mu$ capture on the proton $\left(q_{0} \approx 0 ;|g| \approx m_{\mu}\right)$. The basic mechanism is believed to be well understood as the impulse approximation on free nucleons, so that the final states are determined by standard nuclear physics. Corrections to this picture concern finer details only.

The anomalous experimental results occur when a large part of the muon rest mass has gone into nuclear excitation. of particular interest are the protons, which can have considerable energy ${ }^{2)}$. In the region above evaporation, it is observed that protons above $30 \mathrm{MeV}$ have a $0.2 \%$ yield, for example. Protons of these energies have no place in an impulse picture with uncorrelated nucleons and they demonstrate many-body processes. So do the high energy neutrons 3$), 4)\left(E_{n}>30 \mathrm{MeV}\right)$ with a yield about $1 \%$. These experimental indications hint very clearly at a capture mechanism similar to that in nuclear photo-disintegration and in $\pi$ capture, in which high energy nucleons are emitted.

The impulse approximation for uncorrelated nucleons cannot give even a qualitative description of these phenomena. The failure is probably an important one at larger excitation. For the somewhat similar case of pion capture which corresponds to $140 \mathrm{MeV}$ nuclear excitation, the impulse approximation for uncorrelated nucleons is known to fail 5) by a factor $10^{3}-10^{4}$. Consequently there have been attempts to describe the anomalous $\mu$ capture using correlated nucleon pairs ${ }^{2)}$. While such descriptions contain some truth, it is difficult to obtain reliable and controllable predictions in this way as is well known from $\pi$ capture. Consequently, the nuclear $\mu$ capture phenomenon at large energy transfer is at present a nearly unexplored region theoretically, and only slightly less so experimentally. 
In the present article we will give a quantitative theory for nuclear $\mu$ capture at large excitations. The key point is the deep connection between axial $\mu$ capture and $\pi$ capture. In a previous article ${ }^{6)}$ we have shown that such a connection follows naturally from PCAC on the one hand, and from a locality condition on the axial current on the other hand. From this view point the resemblance of $\mu$ capture processes to pion processes is clear since both are basically the same process. Although our picture allows the discussion of partial $\mu$ capture as a pion process we will here discuss the inclusive capture rate only. For technical simplicity and so as to be specific we have limited ourselves to the case of $\mu$ capture at maximal energy transfer. At this point the $\mu$ capture has $q_{0}=m_{\mu} ;|q|=0$, which is remarkably similar to $\pi$ capture at rest $q_{0}=m_{\pi} ;|g|=0$, and makes a ccmparison particularly interesting. Since it is difficult to use detailed nuclear wave functions in a process of so large energy transfer, it is natural to express the result directly in the known properties of the $\pi$ nuclear interaction; this is the road we will follow.

We first give the formal expression for the $\mu$ capture rate at $q_{0}=m_{\mu},|q|=0$ and show that it separates into three incoherent terms at this point. We then demonstrate that as a rigorous consequence of PCAC alone, the timelike part of the axial $\mu$ capture is given by the $s$ wave pion absorption with $\mathrm{w}_{\pi}=\mathrm{q}_{\mathrm{O}}=\mathrm{m}_{\mu}$. The assumption of axial locality 6 ) is then used to relate the remaining axial capture to pion $p$ wave capture. Since there is also vector capture for muons, we relate this term to the photonuclear cross-section at $\mathrm{w}_{\gamma}=\mathrm{m}_{\mu}$ and show it to be numerically unimportant. We illustrate our conclusions with a numerical example.

$$
\left.i^{-}(i, m)+N(p, M) \rightarrow l_{i}(i, \hat{c})+X\left(P^{\prime}, N\right)_{x}\right)
$$


The notation is (four-momentum, mass) with the four-momentum transfer defined by $q=l-l l$. The final state $X$ corresponds to any possible nuclear channel ; the mass $\mathbb{M}_{X}$ includes also the nuclear excitation energy and relative kinetic energies in this state.

With the standard weak interaction theory of charged currents, the matrix element for the process (1) is

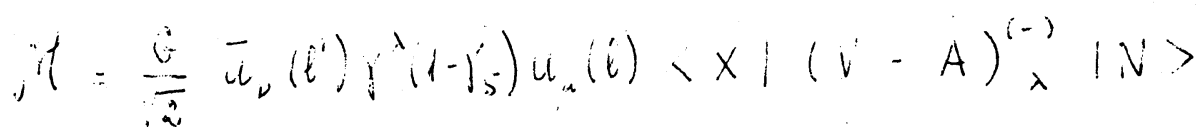

The strangeness conserving currents have the coupling constant $G=G_{F} \cos \theta_{C}$.

The $\mu$ capture from the atomic 1 s orbit and with a neutrino in the energy interval $d E$ is obtained by summing over all available states $X$ compatible with energy momentum conservation. We will in addition sum and average over spins.

For the special case of maximal energy transfer $q_{0}=m_{\mu}$; $|g|=0$ (i.e., $E=0$ ), the sum over matrix elements has several simplifications. First, there are no interference terms between matrix elements in $\underset{\sim}{\sim}, V_{0}, \underset{\sim}{\sim}$ and $\left.A_{0}{ }^{*}\right)$. Secondly, the conservation of the vector current (CVC) has the consequence that all matrix elements in $V_{0}$ vanish. As we approach maximal energy transfer we have therefore

$$
\begin{aligned}
d \Gamma= & \frac{G^{2} E^{2} d E}{2 \pi M}\left|\psi_{i s}(U)\right|^{2} x \\
& \frac{(a-\pi)^{3}}{2} \geq-(q+P-P)\left\{\left|\left\langle x\left|A_{i}^{(-)}\right| N\right\rangle\right|^{2}+\left|\left\langle x\left|A^{(-)}\right| N\right\rangle\right|^{2}+\left|\left\langle x\left|V^{(-)}\right| N\right\rangle\right|^{2}\right\}
\end{aligned}
$$

The capture rate goes to zero with $E^{2}$ in the limit of maximal energy transfer, so that our discussion carries on the quantity between brackets in Eq. (3), which behaves as a constant. We will now study this quantity from a dynamical point of view.

*) The leptonic part weighs them all equally. 


\section{STRICT CONSEQUENCES OF PCAC}

We assume the divergence of the axial current given by the interpolating pion field in the form 7)

$$
\because \pi^{\mu(-)}(x)=f_{\pi} m_{\pi}^{2} \psi^{(-)}(x)
$$

with the charged pion decay constant $f_{\pi}=0.94 \mathrm{~m}_{\pi}$. The matrix element of Eq. (4) between $N$ and $X$ for the special kinematics $q_{0}=m_{\mu}$ and $|q|=0$

$$
\begin{aligned}
& \left\langle\lambda\left|H^{i-)}\right| N\right\rangle=-i \frac{m_{n}^{2}}{q_{i}} f_{\pi}\left\langle x\left|\psi^{(-)}\right| N\right\rangle=-\frac{i}{m_{i}} \frac{m_{n}^{2} f_{\bar{n}}}{m_{n}^{2}-m_{\mu}^{2}}\left\langle x\left|j_{j}^{(-)}\right| N\right\rangle \\
& {\left[q=0, q^{3}=m_{\mu}\right]}
\end{aligned}
$$

Here we have introduced the pion source $j(x)$ defined by $\left(\square+m_{\pi}^{2}\right) \varphi(x)=j(x)$. One notes that the right-hand side of $\mathrm{Eq}_{\perp}$ ( $(5)$ corresponds to an $\underline{s}$ wave pion capture amplitude $(\underset{\sim}{q}=0)$.

We give only the inclusive capture rate. Because of the sum over all final states $X$ the rate is proportional to the elastic absorption amplitude for $s$ wave pions with $w_{\pi}=q_{0}=m_{\mu}$, or in other words, to the off-shell imaginary $s$ wave scattering length

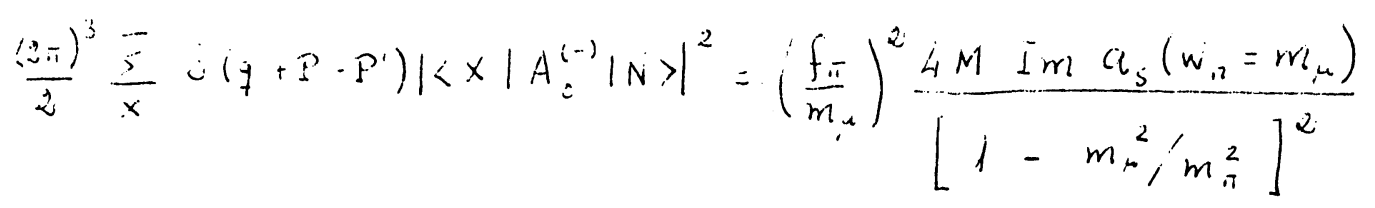

From Eq. (3) we therefore immediately conclude that the capture rate for maximal energy transfer has a strict lower bound given by PCAC :

$$
\left.\frac{d !^{2}}{d E}\right|_{E \rightarrow c} \gg \frac{2 \dot{G}^{2} E^{2}}{\pi}|\psi(c)|^{2}\left[\frac{f_{\pi} m_{\pi}^{2} / m_{\mu}}{m_{\pi}^{2}-m_{\mu}^{2}}\right]^{2} \operatorname{Im} a_{S}\left(w_{\pi}=m_{\mu}\right)_{(7)}
$$

This remarkably simple relation can be interpreted as due to an external pion source proportional to the muon probability : pions are generated from the muon over the nuclear volume with a strength dictated by 
PCAC. Since the source has spherical symmetry for $\underset{\sim}{\sim}=0$, it corresponds to an incident $s$ wave pion of energy $w_{\pi}=m_{\mu}$. In complete analogy to the physical pion, the absorption of this pion is given by the imaginary elastic amplitude at $\mathrm{w}_{\pi}=\mathrm{m}_{\mu}$.

It would be totally wrong to believe that $\mathrm{Eq} .(7)$ is the pion pole contribution, with a strength given by the virtual $\mu \rightarrow \pi \nu$ amplitude and by the on-shell pion absorption. First, according to PCAC the effective strength of the pion source is $\mathrm{m}_{\pi}^{2} / \mathrm{m}_{\mu}$ and not $\mathrm{m}_{\mu}$ as given by the $\mu \rightarrow \pi \nu$ vertex, so that the rate $(7)$ is multiplied by $\left(m_{\pi}^{\mu} / m_{\mu}\right)^{4} \simeq 3$. Secondly, the pion amplitude itself is not the physical one and it may differ radically from it. Even if the nucleus is assumed to be pointlike, it will differ, simply because the pion has an imaginary wave number (unrelated to its momentum) ik with $k=\left(m_{\pi}^{2}-m_{\mu}^{2}\right)^{\frac{1}{2}}$. In the scattering length approximation we have

$$
\operatorname{Im} a_{s}^{p i n t}\left(w_{n}=w_{j}\right)=\frac{\operatorname{Im} a_{s}}{\left|1+k a_{s}\right|^{2}}
$$

The point approximation (8) gives the correct leading term to order $k$ in the $\pi-\mu$ mass difference and it should provide a reasonable first guide for light nuclei with $k R \lesssim 1$. While the amplitude $a_{S}\left(w_{\pi}=m_{\mu}\right)$ is rather closely connected to the physical amplitude in light nuclei, this is not the case for heavy nuclei. Physical pions do not penetrate into the interior of heavy nuclei, but are absorbed on the nuclear surface. On the contrary muons are present everywhere in the nucleus and could produce a volume effect. It is therefore clear that the physical surface absorption of pions cannot correctly describe the volume absorption in Eq. (7). This volume term exists also for pions when $k R \neq 0$. The explanation is that pions are generated inside the nucleus by the muons and that they are absorbed there locally. In this way a volume effect enters naturally. This phenomenon is closely related to coherent propagation of virtual pions in high energy neutrino reactions as has been discussed by Bell 8). The highly interesting physics of the pion amplitude below threshold will be iscussed in a separate publication ${ }^{9}$ ).

We turn now to the two remaining terms in Eq. (3). Since the predictive power of PCAC has been exhausted by its use in Eq. (7), the discussion of the space part of the axial current will require an additional concept. For this we use the idea of locality of the axial current 6 ) which links $\stackrel{A}{\sim}$ to the pion $p$ wave. 
CONSEQUENCE OF AXIAL LOCALITY 6)

If the axial current is decomposed into a pionic field part $\partial_{\mu} \varphi$ and a remaining contribution $\widetilde{A}_{\mu}$ we have

$$
A_{\mu}(x)=-f_{\pi} \partial_{\mu} \dot{\psi}(x)+\tilde{A}_{\mu}(x)
$$

From PCAC it follows that the divergence of $\widetilde{A}_{\mu}$ is proportional to the pionic source

$$
\vartheta_{\mu} \check{A}^{\mu}(x)=f_{\pi} f(x)
$$

At this point it is advantageous to choose a non-covariant notation in the description of the pion, since this makes it easy to separate the source into the local (energy dependent) $s$ and $p$ wave absorption terms :

$$
j\left(q_{i}, \underset{\sim}{x}\right)=S\left(q_{0}, \underset{\sim}{x}\right)-\underset{\sim}{V} \cdot S\left(q_{0}, \underset{\sim}{x}\right)
$$

The two structure functions $s\left(q_{0}, \underset{\sim}{x}\right)$ and $\underset{\sim}{S}\left(q_{0}, \underset{\sim}{x}\right)$ are characteristic local properties of the nucleus at the point $\underset{\sim}{*}$. If we now insist that $\widetilde{A}_{\mu}\left(q_{0}, \mathbb{x}\right)$ depends only on these pionic source terms at the point $\underset{\sim}{\mathbb{X}}$ ("the point $x^{\prime \prime}$ means pointlike on the nuclear scale) this leads immediately to the identification of $\widetilde{A}_{0}$ and $\underset{A}{\sim}$ with the transition density for $s$ and $\mathrm{p}$ wave pions :

$$
\begin{aligned}
& \tilde{A_{c}}\left(q_{0}, \underset{\sim}{x}\right)=-i \frac{f_{\bar{T}}}{q_{0}} s\left(q_{0}, \underset{\sim}{x}\right) \\
& \tilde{A}\left(q_{0}, \lambda\right)=f_{\bar{n}} S_{\sim}^{S}\left(q_{0}, \underset{\sim}{x}\right)
\end{aligned}
$$

This is the statement of "axial locality". The background of Eqs. (12) has been discussed in detail by two of us previously 6)。

For the special case of maximal energy transfer, the matrix element of $\underset{\sim}{\stackrel{\sim}{\sim}(-)}$ is the Fourier transiorm at $\underset{\sim}{\sim}=0$ (volume integral). The content of Eq. (12b) follows then from the weaker assumption that only the time component $\widetilde{A}_{0}$ is given locally ${ }^{6)}$. 
The important point in this context is therefore that $\underset{\sim}{\stackrel{\sim}{\sim}}(-)$ is given by the local pion $p$ wave interaction. For the special case of lighter nuclei $(A \lesssim 30)$ the local pion $p$ wave interaction dominates 10$)$ the $p$ wave scattering lengths : the local $s$ wave interaction is too weak to influence it significantly. We will assume this situation to be valid also below threshold, so that light nuclei are taken to have the matrix element of $\underset{\sim}{\sim}(-)$ related to the global $p$ wave amplitude. We then have

$$
\begin{aligned}
& \frac{(2 \pi)^{3}}{2} \frac{5}{x} j(q+P \cdot P)|: x| A^{(-)}|N>|^{2} \\
& =3 \frac{(2 \pi)^{3}}{2} \frac{5}{x} i\left(q+P-p^{\prime}\right) f_{\pi}^{2}\left|\left\langle x\left|\hat{q} \cdot s^{-1}\right| N\right\rangle\right|^{2} \\
& \approx 9 f_{\pi}^{2} 4 M \operatorname{Im} a_{p}\left(w_{\pi}=w_{n}\right)
\end{aligned}
$$

The first equality in Eq. (13) follows from (12b) and the spatial symmetry at $\underset{\sim}{q}=0$. The last equality expresses that $p$ wave pion absorption summed over all $X$ is related to elastic $p$ wave amplitude by a partial wave unitarity condition. The approximation in this term is the neglect of the local $s$ wave interaction. As in the corresponding relation for the $s$ wave amplitude below threshold, Eq. (6), the relation (13) does not contain the physical amplitude. Even for a pointlike situation one has

$$
\operatorname{im} a_{p}^{p i n t}\left(w_{i}=m_{\mu}\right)=\frac{\operatorname{Im} a_{p}}{\left|1-k^{3} a_{p}\right|^{2}}
$$

where $a_{p}$ is the physical $\pi$ nuclear scattering volume and $k=\left(m_{\pi}^{2}-m_{\mu}^{2}\right)^{\frac{1}{2}}$. The simple approximations (13) and (14) are not valid in a heavy nucleus. In this case it is important to insist on the local $p$ wave interaction in the medium. In addition, the question of volume absorption vs. surface absorption appears once more in this case with essentially the same explanation. There exists a well defined procedure for a discussion of absorption in this case using the $\pi$ nuclear optical potential description ${ }^{9}$ ). 
An estimate of the contribution of the vector current in $\mathrm{Eq}$. (3) can be obtained from the physical photo-absorption cross-sections on nuclei. Consider the physical photon process

$$
y(q)+N(p) \rightarrow X\left(p^{\prime}\right)
$$

with $q^{2}=0$, i.e., $|\underset{\sim}{q}|=q_{0}$. The unpolarized absorption cross-section is given by

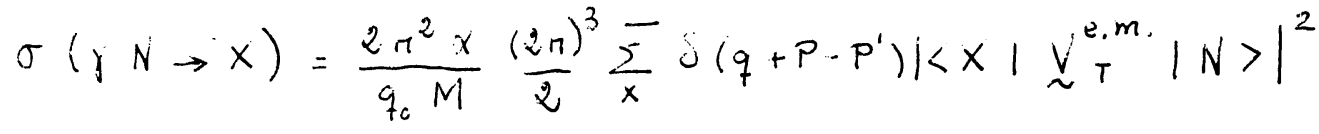

$$
\begin{aligned}
& {\left[|\stackrel{q}{\sim}|=q_{0}\right]}
\end{aligned}
$$

where $\underset{\sim}{V_{T}^{e} \cdot m}$. is the transverse electromagnetic current, which contains both isovector and isoscalar contributions.

We are interested in matrix elements of the weak vector current for $\underset{\sim}{q}=0$, however. The use of CVC tells us that there is no isoscalar contribution for matrix elements with $\underset{\sim}{q}=0$. In addition, according to the strong version of $\mathrm{CVC}$, the isovector electromagnetic and weak currents are components of the same isospin vector. Therefore

$$
\begin{aligned}
& \frac{(2 n)^{3}}{2} \sum \bar{c}\left(q+P-P^{\prime}\right)\left|\left\langle\times\left|v_{n}^{(-)}\right| N\right\rangle\right|^{2} \\
& =\frac{3}{2}(2 n)^{3} \sum \dot{\partial}\left(q+P-P^{\prime}\right)\left|\left\langle x\left|v_{m}^{\prime e} T\right| N\right\rangle\right|^{2} \\
& {[q=0]}
\end{aligned}
$$

The right-hand side of Eq. (17) corresponds to unphysical matrix element of the electromagnetic current. The vector currents in Eqs. (16) and (17) have therefore different $\underset{\sim}{\sim}$ values, but the same value of $q_{0}$. We now discuss the problem of the extrapolation in $\underset{\sim}{q}$.

For photon energies above $50 \mathrm{MeV}$, total photonuclear absorption cross-sections are given empirically by 11)

$$
\sigma_{\gamma}=28 \gamma \frac{N Z}{A} \frac{\left(E_{\gamma}-2.225\right)^{3 / 2}}{E_{\gamma}^{3}}(m b)
$$


where $E_{\gamma}$ is the photon energy in $\mathrm{MeV}$. The empirical dependence with any specific nucleus in $\mathrm{Eq}$. (18) only enters by the number of neutrons and protons. There is no trace of any form factor in $q^{2}$ related to the variable size of different nuclei. The result (18) is therefore described as photoabsorption by incoherent subsystems in nucleus (quasi-deuteron model). The subentities will act incoherently for distances larger than the scale $r_{0}$ of the quasi-deuteron process in reactions which are summed over all final states. The relevant momentum transfer is therefore not $R^{-1}$, the inverse of the nuclear radius, but $r_{0}^{-1}$, the scale of the quasi-deuteron process. Consequently it will be an excellent approximation to use the long wave length limit for the electromagnetic current in inclusive processes (this does not mean that high multipoles and retardation do not contribute in partial channel photoreactions, but that there is a sum rule !). Since the unretarded matrix element $(q=0)$, for fixed $q_{0}$, is precisely the one we need in Eqs. (3) and (17) for the weak vector current, we have the relation

$$
\frac{(2 \pi)^{3}}{2} \sum_{x} \delta\left(q+p-p^{\prime}\right)\left|\left\langle x\left|\underline{v}^{(-)}\right| N\right\rangle\right|^{2} \approx \frac{3 m_{\mu} M \mid}{2 \pi^{2} x} J_{\gamma}\left(E_{\gamma}=m_{\mu}\right)
$$

\section{FINAL EXPRESSION AND NUMERICAL APPLICATION}

The $\mu$ capture rate near maximal energy transfer is obtained from the partial results (6), (13) and (19)

$\left.\frac{d \Gamma}{d E}\right|_{E \rightarrow 0}=\frac{2 G^{2} E^{2}}{\pi}\left|\psi_{1 S}(0)\right|^{2}\left\{f_{\pi}^{2}\left[\left(\frac{m_{\pi}^{2}}{m_{\mu}}\right)^{2} \frac{j m a_{S}\left(w_{12}=m_{\mu}\right)}{\left[m_{\pi}^{2}-m_{\mu}^{2}\right]^{2}}+9 I m a_{p}\left(w_{\pi}=w_{\mu}\right)\right]+\frac{3 m_{\mu}}{8 i T^{2} \cdot x} \sigma_{r}\left(E_{r}=m_{\mu}\right)\right\}$

To illustrate the relative importance of the different terms in a practical case we choose the example of ${ }^{12} \mathrm{C}$. This is a light nucleus for which the point approximation to the $\pi$ nuclear $s$ and $p$ wave amplitudes is expected to be a satisfactory guide. The physical threshold amplitudes for $\pi{ }^{12} \mathrm{C}$ are 12$), *$ )

*) We thank Dr。I. Tauscher for the $p$ wave values. The imaginary part of $a_{p}$ reproduces the observed $\Gamma_{2 p}$ in the $\pi 1{ }^{1} \mathrm{C}$ atom, while the real part is the theoretically expected value using best optical model parameters. 


$$
a_{s}=(-.32+i .10) m_{i}^{-1}, \quad a_{p}=(.72+i .14) m_{m}^{-3}
$$

For a point system the unitarity corrections (8) and (14) are well defined. For the case of ${ }^{12} \mathrm{C}$ they increase both the $s$ and $p$ wave amplitudes at $w_{\pi}=m_{\mu}$ by about $40 \%$ as compared to the physical amplitudes. For heavier nuclei the extrapolation below threshold is more intricate. It is well defined, however, and amounts in essence to the solution of a wave equation with a potential and a driving source [see the previous discussion and Ref。9

The experimental photoabsorption cross-section for ${ }^{12} \mathrm{C}$ at $\mathrm{E}_{\gamma}=106 \mathrm{MeV}$ is experimentally $\left.\sigma_{\gamma} \approx 1 \mathrm{mb} 11\right)$. With these input data we have for ${ }^{12} \mathrm{C}$ with $\epsilon=\mathrm{E} / \mathrm{m}_{\mu}$

$$
\frac{d \Gamma}{d \epsilon}=(3.9+5.9+0.8) \epsilon^{2} 10^{4} \sec ^{-1} \approx \epsilon^{2} 10^{5} \mathrm{sec}^{-1}
$$

The experimental total $\mu$ capture rate for ${ }^{12} \mathrm{C}$ is $\left.{ }^{13}\right) \quad \Gamma=3.7 \times 10^{+4} \mathrm{sec}^{-1}$. The total capture yield with an energy transfer from $\left(m_{\mu}-E\right)$ to $m_{\mu}$ is obtained by integrating Eq. (22) for neutrino energies from 0 to $E$. It is approximately

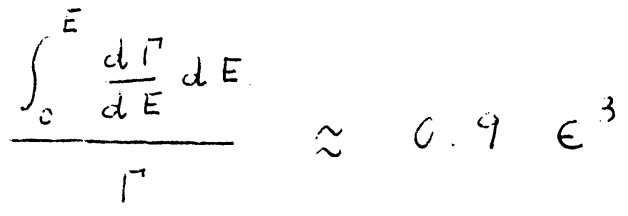

We can now conclude the following :

i) As previously discussed the first term in Eqs. (20) and (22) gives a rigorous lower limit, limited only by the uncertainty in the knowledge of $a_{S}\left(w_{\pi}=m_{\mu}\right)$. This limit is about $40 \%$ of the rate we expect including the additional assumption of "axial locality".

ii) The vector current plays only a marginal rôle in the large energy transfer $\mu$ capture $(\lesssim 10 \%)$. The physics is therefore nearly completely dominated by the axial current and consequently by pion physics. 
iii) The timelike and the spacelike parts of the axial current contribute about equally to the capture rate, so that both $s$ and $p$ wave pion amplitudes must be carefully taken into account. The importance of the time component contrasts sharply with the usual low energy transfer region with $|\stackrel{A}{A}|^{2} \gg\left|A_{0}\right|^{2}$.

iv) The present picture leads to a surprisingly strong rate for large energy transfer capture : from Eq. (23) about 1\% of the capture should have an energy transfer of more than $80 \mathrm{MeV}$. This explains qualitatively the energetic neutrons and protons in $\mu$ capture : if we assume that two fast nucleons are typically emitted in a $\mu$ capture as for $\pi$ capture, about $15-20 \mathrm{MeV}$ will go into binding energy and residual excitation. The typical energy of a nucleon emitted after $80 \mathrm{MeV}$ energy transfer is then about $30 \mathrm{MeV}$. This agrees with the experimental observation of about $1 \%$ capture into neutrons of more than $30 \mathrm{MeV}^{3)}$.

v) The previous conclusion means that there are strong reasons to believe correlated nucleons to be emitted in $\mu$ capture with substantial probability. Their experimental observation would provide a much better test of the present picture.

To summarize : we have introduced a novel and appealing description of $\mu$ capture at large energy transfer with no free parameter. Contrary to the usual approach we do not appeal to $\mu$ capture on individual nucleons and we do not require any detailed knowledge of nuclear wave functions. Instead we have quantitatively transformed the problem into a problem of nuclear pion interactions below threshold. The basic ingredients for this connection were PCAC on the one hand and the idea of "axial locality" on the other. Our picture is qualitatively in agreement with experimental indications of processes in the large energy transfer region, and it leads for instance to the prediction of two-nucleon $\mu$ capture process.

The present approach draws attention to some additional problems which merit further investigation and discussion.

Partial capture rates. The partial capture amplitudes link also directly to pionic processes, but this aspect remains to be investigated in more detail. One notes in particular that the two-nucleon capture is expected to play a prominent rôle. 
$\mu$ capture at other energy transfers. If "axial locality" is strictly applied, it predicts that all axial $\mu$ capture is described by pions. Excellent arguments for such a connection exist at low energy transfer 14), but no quantitative studies exist for intermediate energy transfer.

Physics of subthreshold pions. The present picture makes it necessary to understand the physics of the nuclear pion field below the physical region $\mathrm{w}_{\pi}=\mathrm{m}_{\pi}$ and its relation to the off-mass shell scattering amplitudes in this region. In particular, it is of special importance to achieve an understanding of the dependence on the nuclear size, on the $\mu \pi$ mass difference as well as on the strength of the $\pi$ nuclear interaction. As briefly indicated in the text, the behaviour is a non-trivial one with new physics. In particular, tine surface interaction of the physical amplitude for a large system becomes a volume interaction below threshold in the muonic problem ${ }^{9}$ ). 


\section{REFERENCES}

1) See, for example, the review article by N. Mukhopadhyay - Physics Reports, to be published.

2) Yu.G. Budyashov et al. - JETP 33, 11 (1971);

Yu.A. Batusov and R.A. Eramzhyan - Dubna Report E1-9457 (1976).

3) R.M. Sundelin and R.M. Edelstein - Phys.Rev. C7, 1037 (1973).

4) P. Singer - Springer Tracts in Modern Phys. 71, 39 (1974).

5) J. Le Tourneux - Nuclear Phys. 81, 665 (1966).

6) T.E.O. Ericson and J. Bernabeu - CERN Preprint TH 2304 (1977).

7) Y. Nambu - Phys.Rev.Letters 4, 380 (1960) ;

M. Gell-Mann and M. Lévy - Nuovo Cimento 16, 705 (1960).

8) J.S. Bell - CERN Preprint TH 887 (1968).

9) J. Bernabéu and T.E.O. Ericson - To be published;

T. Ericson - "Somewhat Virtual Pions", Lectures at the International School on "Interface Between Particle Physics and Nuclei", Erice (September 1976). CERN Preprint TH 2257 (1976).

10) M. Krell and T.E.O. Fricson - Nuclear Phys. B11, 521 (1969).

11) J.Ahrens et al. - Nuclear Phys. A251, 479 (1975) ;

B. Ziegler - MPI Mainz Nuclear Physics Internal Note (1976).

12) J. Hüfner, I. Tauscher and C. Wilkin - Nuclear Phys. A231, 455 (1974).

13) M. Eckause, R.T. Siegel, R.E. Welsh and T.A. Filippas - Nuclear Phys. 81, $575(1966)$.

14) J. Delorme, M. Ericson, A. Figureau and C. Thévenet - Ann.Phys. (N.Y.) 102, 273 (1976). 